\title{
Measurement of the transverse target-spin asymmetry associated with deeply virtual Compton scattering on the proton
}

\author{
Zhenyu Ye ${ }^{* \dagger}$ \\ DESY, 22607 Hamburg, Germany \\ E-mail: yezhenyu@mail.desy.de
}

\begin{abstract}
Measurements of deeply virtual Compton scattering (DVCS) made at HERMES using $27.6 \mathrm{GeV}$ $e^{ \pm}$beams and various internal polarized or unpolarized gaseous targets are discussed. Results are reported on the transverse target-spin asymmetry (TTSA) associated with DVCS, extracted from data accumulated in 2002-2004 with the $e^{+}$beam on a transversely polarized hydrogen target. TTSA amplitudes leading in twist and $\alpha_{S}$ are given as a function of $-t, x_{B}$ and $Q^{2}$ in the kinematic range $|t|<0.7 \mathrm{GeV}^{2}, 0.03<x_{B}<0.35$ and $1<Q^{2}<10 \mathrm{GeV}^{2}$. Theoretical predictions based on a phenomenological model of generalized parton distributions (GPDs) agree with the experimental results. With additional statistics accumulated in 2005 , one may constrain the $u$-quark total angular momentum in the nucleon within this model.
\end{abstract}

International Europhysics Conference on High Energy Physics

July 21st - 27th 2005

Lisboa, Portugal

*Speaker.

${ }^{\dagger}$ on behalf of the HERMES collaboration 


\section{Introduction}

Deeply virtual Compton scattering (DVCS) is an exclusive process in which a virtual photon (emitted by an incoming lepton) is absorbed and a real photon is produced by a single parton in the nucleon, the recoiling nucleon being in its ground state. It is one of the theoretically cleanest processes to access generalized parton distributions (GPDs); the GPDs provide a detailed description of the nucleon structure. Strong interest in DVCS arose recently after it was realized that GPDs encode information about the total angular momentum of the partons in the nucleon [1].

The final state of DVCS is identical to that of the Bethe-Heitler (BH) process, in the latter the real photon being radiated from the incoming or scattered lepton. Hence the experimental cross section contains the interference between the BH amplitude $\mathscr{T}_{B H}$ and the DVCS amplitude $\mathscr{T}_{D V C S}$ :

$$
\frac{d \sigma}{d x_{B} d Q^{2} d|t| d \phi d \phi_{S}} \propto\left|\mathscr{T}_{B H}\right|^{2}+\left|\mathscr{T}_{D V C S}\right|^{2}+\mathscr{T}_{B H}^{*} \mathscr{T}_{D V C S}+\mathscr{T}_{B H} \mathscr{T}_{D V C S}^{*}
$$

Here $x_{B}$ denotes the Bjorken scaling variable, $-Q^{2}$ the square of the 4-momentum of the virtual photon and $t$ the square of the 4-momentum transfer between initial and final target nucleons. The angle $\phi\left(\phi_{S}\right)$ denotes the azimuthal angle of the photon production plane (the target polarization vector) with respect to the lepton scattering plane, measured around the direction of the virtual photon (see figure 1).

While $\mathscr{T}_{D V C S}$ contains information about unknown GPDs, $\mathscr{T}_{B H}$ is exactly calculable in QED using the knowledge of the well-determined nucleon form factors [2]. In fixed-target experiments at moderate $Q^{2}$ (e.g. at HERMES [3]), where the BH contribution widely dominates the cross section, DVCS results have already been obtained by measuring the azimuthal asymmetry in the cross section with respect to the beam helicity [4], to the beam charge [5], and to the longitudinal target-spin orientation [6]. In this article we will report the first results on the transverse target-spin asymmetry (TTSA) obtained at HERMES.

\section{The HERMES Experiment}

HERMES studies the spin structure of the nucleon using the $27.6 \mathrm{GeV}$ electron (or positron) beam at HERA and internal polarized $\mathrm{H}, \mathrm{D}$, and ${ }^{3} \mathrm{He}$ gaseous targets. A spectrometer magnet

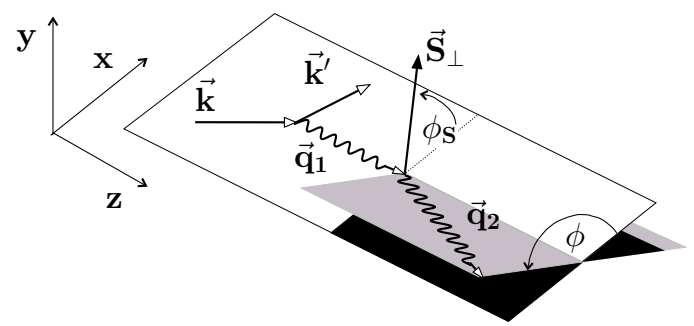

Figure 1: Kinematics and azimuthal angles of real photon electroproduction in the target rest frame. The $z$-direction is chosen along the three-momentum of the virtual photon $\vec{q}_{1}$. The lepton three-momenta $\vec{k}$ and $\vec{k}^{\prime}$ form the lepton scattering plane, while the three-momenta of virtual and real photons $\vec{q}_{1}$ and $\vec{q}_{2}$ define the photon production plane. The azimuthal angles $\phi$ and $\phi_{S}$ are explained in the text. 
instrumented with tracking chambers provides good momentum $(\delta p / p<2 \%)$ and angular $(\delta \theta<1$ mrad) measurements for charged particles [3]. Good lepton-hadron separation is achieved by a transition-radiation detector, a pre-shower counter and an electromagnetic calorimeter, which also detects the photon.

Not all the hadrons in the final state of the BH and DVCS processes are detected at HERMES by its forward spectrometer - the recoiling proton typically travels perpendicularly to the beam direction and hence escapes the detector acceptance. The exclusivity of the selected events, which contain an identified scattered lepton and a produced real photon, is maintained by a missing-mass cut of $-(1.5)^{2}<M_{x}^{2}<(1.7)^{2} \mathrm{GeV}^{2}$. Monte Carlo studies have shown that the non-DVCS contributions to the selected data sample originates mainly from semi-inclusive $\pi^{0}$ production amounting to approximately $5 \%$ [7].

\section{Transverse Target-Spin Asymmetry}

The transverse target-spin asymmetry, measured using an unpolarized (U) lepton beam and a transversely $(\mathrm{T})$ polarized target, is defined as:

$$
A_{U T}\left(\phi, \phi_{S}\right)=\frac{d \sigma\left(\phi, \phi_{S}\right)-d \sigma\left(\phi, \phi_{S}+\pi\right)}{d \sigma\left(\phi, \phi_{S}\right)+d \sigma\left(\phi, \phi_{S}+\pi\right)} .
$$

The two azimuthal amplitudes $A_{U T}^{\sin \left(\phi-\phi_{S}\right) \cos \phi}$ and $A_{U T}^{\cos \left(\phi-\phi_{S}\right) \sin \phi}$, which are associated with DVCS on the proton, can be approximated in leading twist and $\alpha_{S}$ as [8]:

$$
\begin{aligned}
A_{U T}^{\sin \left(\phi-\phi_{S}\right) \cos \phi} & \propto \pm f\left(x_{B}, Q^{2}, t\right) \cdot \operatorname{Im}\left[F_{2} \mathscr{H}-F_{1} \mathscr{E}\right], \\
A_{U T}^{\cos \left(\phi-\phi_{S}\right) \sin \phi} & \propto \pm f\left(x_{B}, Q^{2}, t\right) \cdot \operatorname{Im}\left[F_{2} \widetilde{\mathscr{H}}-F_{1} \xi \widetilde{\mathscr{E}}\right] .
\end{aligned}
$$

Here $+(-)$ stands for a negatively (positively) charged lepton beam and $f\left(x_{B}, Q^{2}, t\right)$ denotes a kinematic factor that is independent on the azimuthal angles. $\mathscr{H}, \mathscr{E}, \widetilde{\mathscr{H}}$ and $\widetilde{\mathscr{E}}$ denote Compton form factors which are convolutions of the respective twist-2 GPDs $H, E, \widetilde{H}$ and $\widetilde{E}$ [2]. $F_{1}$ and $F_{2}$ are the Dirac and Pauli form factors of the proton, respectively. Note that dependences on kinematic variables are partially omitted in equation (3.2) for clarity.

Figure 2 shows $A_{U T}^{\sin \left(\phi-\phi_{S}\right) \cos \phi}$ and $A_{U T}^{\cos \left(\phi-\phi_{S}\right) \sin \phi}$ as a function of $-t, x_{B}$ and $Q^{2}$, as extracted at HERMES. The analysis is based on data accumulated in 2002-2004 with the positron beam and a transversely polarized hydrogen target. Corrections for background and smearing have been applied. The main contributions to the systematic uncertainty are those from the determination of the target polarization and in the background correction. Also shown in figure 2 are theoretical predictions based on a phenomenological model of GPDs [9]. The curves represent the TTSA amplitudes evaluated with different $u$-quark total angular momentum $J_{u}$ as a model parameter, while fixing the $d$-quark total angular momentum $J_{d}=0$ [8]. The experimental results are in general agreement with the theoretical predictions. More precise asymmetries could clearly distinguish between predictions with different $J_{u}$, within this model.

\section{Summary and Outlook}

The transverse target-spin asymmetry associated with DVCS on the proton has been measured at HERMES. An investigation [8] has shown that a measurement of the TTSA associated with 


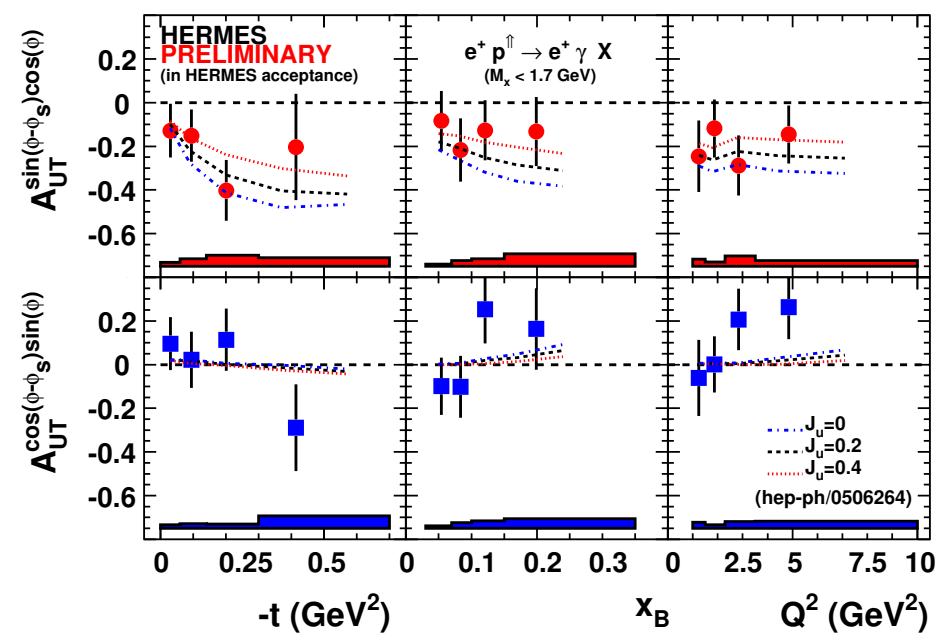

Figure 2: The $A_{U T}^{\sin \left(\phi-\phi_{S}\right) \cos \phi}$ and $A_{U T}^{\cos \left(\phi-\phi_{S}\right) \sin \phi}$ amplitudes of the transverse target-spin asymmetry associated with DVCS, shown as a function of $-t, x_{B}$ and $Q^{2}$ for the exclusive sample $\left(-(1.5)^{2}<M_{x}^{2}<(1.7)^{2}\right.$ $\mathrm{GeV}^{2}$ ) after background correction. The error bars (bands) represent the statistical (systematic) uncertainties. The curves are the predictions from a GPD model with different $u$-quark total angular momentum $J_{u}$ and fixed $d$-quark total angular momentum $J_{d}=0[8]$.

DVCS could lead to constraint of the $u$-quark total angular momentum in the nucleon within certain GPD models. Such an analysis is in progress, which will include the data accumulated at HERMES in 2005 .

\section{References}

[1] X. Ji, Gauge-Invariant Decomposition of Nucleon Spin, Phys. Rev. Lett. 78 (1997) 610.

[2] See for example, A. V. Belitsky, D. Müller and A. Kirchner, Theory of deeply virtual Compton scattering on the nucleon, Nucl. Phys. B 629 (2002) 323.

[3] K. Ackerstaff et al., The HERMES Spectrometer, Nucl. Instr. and Meth. A 417 (1998) 230.

[4] A. Airapetian et al., Measurement of the Beam-Spin Azimuthal Asymmetry Associated with Deeply-Virtual Compton Scattering, Phys. Rev. Lett. 87 (2001) 182001.

[5] F. Ellinghaus, New Results on Deeply Virtual Compton Scattering at H1, ZEUS and HERMES, in proceedings of International Conference On High Energy Physics 2004 [hep-ex / 0410094 ].

[6] M. Kopytin, Measurement of Deeply Virtual Compton Scattering at HERMES, in proceedings of 13th International Workshop on Deep Inelastic Scattering and QCD (DIS 2005).

[7] B. Krauss, Deeply Virtual Compton Scattering and the HERMES-Recoil Detector, Ph.D thesis, Physikalisches Institut II, FAU Erlangen-Nünberg, 2005.

[8] F. Ellinghaus, W.-D. Nowak, A.V. Vinnikov and Z. Ye, Can the total angular momentum of u-quarks in the nucleon be accessed at HERMES? [hep-ph/0506264].

[9] K. Goeke, M. V. Polyakov and M. Vanderhaeghen, Hard exclusive reactions and the structure of hadrons, Prog. Part. Nucl. Phys. 47 (2001) 401. 